\title{
How to Characterize Disorder
}

\author{
T. Egami \\ Joint-Institute for Neutron Sciences, Department of Materials Science and Engineering and \\ Department of Physics and Astronomy, University of Tennessee, Knoxville, TN 37996 \\ Materials Science and Technology Division, Oak Ridge National Laboratory, \\ Oak Ridge, TN 37831
}

\begin{abstract}
Researchers working on nuclear materials encounter disorder in the atomic structure all the time, usually caused by irradiation. The nature of disorder varies widely, from lattice defects to amorphous phase formation. Generally it is not easy to characterize the state of disorder with accuracy necessary to elucidate the properties caused by structural disorder. However, owing to advances in the tools of characterization and rapid rise in computer power, significant progress has been made in characterizing structural disorder. We discuss how to describe and determine the structure and dynamics of disordered materials using scattering measurements and modeling. Lattice defects caused by irradiation usually has negative effects on properties, but glasses and highly disordered materials can be irradiation resistant, and could be useful as nuclear materials. Characterizing and controlling disorder is becoming an important endeavor in the field of nuclear materials.
\end{abstract}

key words: disorder, lattice defects, amorphous state, irradiation effects 


\section{Introduction}

We know very well how to characterize the structure of crystals, for instance by diffraction experiments. However, a large part of materials we use are disordered, and even noncrystalline. When we face disordered materials we are much less optimistic in our capacity to characterize the structure, because the conventional methods of structural characterization do not work well. In fact just to describe the structure is difficult, because the terms such as "amorphous" or "disorder" provide only negative description. However, owing to recent advances in experimental tools, such as the advent of synchrotron-based radiation sources, and rapid growth in computing power, it is now possible to characterize the structure of disordered materials with considerable accuracy. In this article we discuss how to determine the structure and dynamics of highly disordered materials by scattering experiments, and how to describe disorder in the structure using newly introduced concepts, such as the atomic-level stresses.

In nuclear materials disorder is usually caused by irradiation. When a material is heavily irradiated at low temperatures it often becomes amorphous. We call it amorphous, rather than glass, because there is a subtle but important difference between a glass and an amorphous solid. A glass has a structure which was once in equilibrium at the temperature when the glass was frozen from a liquid. This temperature is called the fictive temperature, $T_{f}$, and characterizes the glassy state [1]. When a liquid is cooled very rapidly the structure becomes frozen, or arrested, at a relatively high temperature. So such a glass has a higher value of $T_{f}$, while a glass slowly cooled has a lower $T_{f}$. When a glass with a high $T_{f}$ is annealed below the glass transition temperature, $T_{g}$, the structure relaxes and $T_{f}$ is lowered. This process is called structure relaxation [2]. On the other hand if a glass-like solid does not have a well-defined fictive temperature, we call this solid amorphous. A solid classified as amorphous can be either highly defective nano-crystals or a glass with strongly heterogeneous local fictive temperatures.

Disorder caused by irradiation damage is usually harmful to the material. But it appears that materials with high degrees of structural and chemical disorder, such as glasses, may be more irradiation resistant, and thus certain types of disorder may be beneficial to the performance of nuclear material. In either case characterizing disorder with high accuracy will greatly facilitate research on nuclear materials.

We first describe the methods of determining the structure by diffraction and dynamics by inelastic scattering. However, the information obtained by scattering is about the two-body 

correlations. In order to elucidate the properties in terms of the structure computer modeling is becoming more popular. It is now relatively easy to perform simulation, such as molecular dynamics (MD) simulation, using widely available software packages. Unfortunately usually the analysis is done still at a relatively rudimental level. In order to describe and understand the models in a more effective and physically meaningful manner we introduce the concept of the local atomic structure and its description in terms of the atomic-level stresses which more directly connects the local structure to various properties.

\section{Structural Characterization by Diffraction}

The structure of a crystal is defined by lattice symmetry, the lattice constants, and a few atomic position parameters, which can be determined by diffraction experiments through the position and intensity of the Bragg peaks [3]. Any non-periodic components, such as structural disorder or thermal atomic vibration, produce diffuse scattering. Certain consequences of irradiation damage, such as local strains, can be evaluated relatively easily from careful measurement of the position and width of the Bragg peaks [3]. However, other damages at the atomic-level have to be evaluated through the study of the diffuse scattering, which is a more difficult undertaking [3]. In fact in the crystallographic structural analysis diffuse scattering is usually discarded as "background".

Liquids and glasses yield no Bragg peaks upon diffraction, and show only diffuse scattering. Therefore the diffraction measurement for liquids and glasses is quite demanding and more involved. Firstly the real background due to scattering not coming from the sample has to be accurately determined and subtracted from the data. Secondly in order to cover a wide range in the momentum transfer, $Q=|\boldsymbol{Q}|, \boldsymbol{Q}=\boldsymbol{k}_{\boldsymbol{i}}-\boldsymbol{k}_{\boldsymbol{f}}$, where $\boldsymbol{k}_{\boldsymbol{i}}$ and $\boldsymbol{k}_{\boldsymbol{f}}$ are the momenta of the probing particle before and after the scattering, a probe with high energy has to be used as discussed below. Thirdly, rigorous data reduction, including absorption correction and correction for incoherent scattering, has to be performed to obtain reliable results [4].

Fortunately the software for data reduction is widely available and the procedure for the measurement is well-established at the synchrotron x-ray facilities or at the pulse neutron facilities [4], so a user can obtain good results by applying for the beamtime to these facilities. 
Through such experiments one obtains the structure function, $S(Q)$, which is normalized to unity at large $Q$. The Fourier-transform of $S(Q)$ is the atomic pair-density function (PDF),

$$
\rho_{0} g(r)=\rho_{0}+\frac{1}{2 \pi^{2} r} \int_{0}^{\infty}[S(Q)-1] \sin (Q r) Q d Q
$$

where $\rho_{0}$ is atomic number density [3,4]. Examples of $S(Q)$ and PDF are shown in Fig. 1 [5]. In eq. (1) the integration has to be carried out to $Q \rightarrow \infty$, but in reality $Q<Q_{\max }=2 k=4 \pi / \lambda$, because $Q=2 k \sin \theta=4 \pi \sin \theta / \lambda$, where $k=\left|\boldsymbol{k}_{\boldsymbol{i}}\right|=\left|\boldsymbol{k}_{f}\right|$ for elastic scattering, $\lambda$ is the wavelength of the probe, and $\theta$ is the diffraction angle. Therefore if $Q_{\max }$ is too small, high- $Q$ portion of the data is left out in evaluating the PDF. This results in so-called termination error, and makes the PDF inaccurate [3,4]. Normally we need to choose the value of $Q_{\max }$ to be $30-35 \AA^{-1}$, in order to suppress termination error. For x-rays this limit corresponds to the x-ray energy of $30-35$ $\mathrm{keV}$ (wavelength of $0.35-0.41 \AA$ ). Therefore the laboratory $\mathrm{x}$-ray sources are totally inadequate; the energy of $\mathrm{Cu} K_{\alpha}$ radiation is only $8 \mathrm{keV}$, and that of Mo $K_{\alpha}$ radiation is $17.4 \mathrm{keV}$. The use of synchrotron radiation is absolute necessity. By using $\mathrm{x}$-rays with the energy over 100 $\mathrm{keV}$ and a two-dimensional detector the PDF can be determined with accuracy in a short time $[4,6]$. For neutron scattering the value of $Q_{\max }$ for thermal neutrons is about $12 \AA^{-1}$, so we have to use a synchrotron-based pulsed neutron source which provides ample intensity of epithermal neutrons with higher energies. The advent of the synchrotron-based radiation sources was crucial in transforming the PDF method from a semi-quantitative tool to a highly accurate experimental method. The data can be readily processed using software such as PDFgetX [7] and PDFgetN [8], which can be downloaded from the source and are available at the facilities capable of obtaining the PDF.

Prior to this transformation of the PDF technique owing to synchrotron-based radiation sources, the method of extended X-ray absorption fine structure (EXAFS) was widely used as a local structural probe $[9,10]$. In the EXAFS method structural information is obtained through the diffraction of internally emitted photo-electrons excited by $\mathrm{x}$-ray which produces oscillations in the energy dependence of the x-ray absorption coefficient. However, to interpret the EXAFS pattern, including the phase-shifts, we have to calculate the dynamics of photo-electrons by 
quantum-mechanical computation. Usually the electron-electron interaction is approximated using the density functional theory (DFT), but inelastic scattering and multiple scattering cannot be accurately estimated. Thus scattering from randomly displaced atoms is strongly attenuated. Consequently the coordination number (the number of the nearest neighbor atoms) in disordered system is underestimated in the EXAFS, and the information on the structure beyond the first nearest neighbors is strongly suppressed and not reliable. In contrast scattering of x-ray and neutron is weak enough to be accurately described by the Born approximation [3,4]. Thus the PDF can be determined up $200 \AA$ or more depending on the $Q$ resolution, allowing the determination of the short-range as well as medium-range structure (Fig. 2) [11]. Because the EXAFS measurement requires synchrotron radiation anyway, it is generally more advisable to use the PDF method than the EXAFS.

The PDF provides only the information on the two-body correlation, whereas properties often depend on correlations with higher order. Nevertheless because the PDF can now be determined with high accuracy it is possible to garner some critical information from the PDF. For instance it is possible to detect small changes in the PDF due to structure relaxation $[12,13]$, and evaluate changes in the fictive temperature due to processing, such as deformation, from small changes in the PDF [14].

A glass is usually isotropic in structure, but if it is under stress it becomes anisotropic. In such a case $S(\boldsymbol{Q})$ and $g(\boldsymbol{r})$ can be expanded by the spherical harmonics [4,15];

$$
S(\boldsymbol{Q})=\sum_{\ell, m} S_{\ell}^{m}(Q) Y_{\ell}^{m}\left(\frac{\boldsymbol{Q}}{Q}\right), \quad g(\boldsymbol{r})=\sum_{\ell, m} g_{\ell}^{m}(r) Y_{\ell}^{m}\left(\frac{\boldsymbol{r}}{r}\right)
$$

where $Y_{\ell}^{\mathrm{m}}(\boldsymbol{x})$ are the spherical harmonics. They are connected through,

$$
g_{\ell}^{m}(r)=\frac{i^{\ell}}{2 \pi^{2} \rho_{0}} \int S_{\ell}^{m}(Q) J_{\ell}(Q r) Q^{2} d Q
$$


where $J_{\ell}(x)$ is the spherical Bessel function. Because $J_{0}(x)=\sin x / x$, eq. (3) for $\ell=0$ is reduced to eq. (1). This approach has been very effective in finding the nature of elasticity and anelasticity in metallic glasses as shown in Fig. 3 [15-17].

\section{Dynamics of Disordered Matter}

In crystalline solids the elementary excitations of lattice and spin are phonons and magnons, which are propagating waves. They can be detected by inelastic scattering measurements of neutrons or X-rays, which yields the dynamic structure factor, $S(Q, E)$, where $\mathrm{E}$ is the energy loss/gain by scattering [18]. Phonons and magnons show well-defined dispersion curves in $S(\boldsymbol{Q}, E)$. In disordered matter they are scattered by disorder in the structure, and their

lifetime becomes short and some of them are localized. In high-temperature liquids most of phonons are overdamped and marginalized. Instead of phonons the elementary excitations are local topological excitations of cutting and forming atomic bonds [19]. To observe such local excitations the conventional approach in inelastic scattering is no longer useful, because excitations are diffuse both in $Q$ and $E$ space. An alternative is to Fourier-transform $S(Q, E)$ to real space and obtain the dynamic PDF (DPDF) [4,20,21],

$$
g(r, E)=\rho_{0}+\frac{1}{2 \pi^{2} \rho_{0} r} \int_{0}^{\infty} S(Q, E) \sin (Q r) Q d Q
$$

or the Van Hove function [22],

$$
g(r, t)=\int g(r, E) \exp \left(-i \frac{E}{\hbar} t\right) d E
$$

With a reactor neutron source inelastic neutron scattering measurements are usually done with a single detector. Thus they are long and tedious experiments, and it is impossible to determine $S(\boldsymbol{Q}, E)$ over a wide range of $Q$ and $E$ in a reasonable time. However, with the pulsed neutron source the data on $S(\boldsymbol{Q}, E)$ are collected in parallel by position sensitive detectors with a large number of pixels. Therefore it is now possible to determine the DPDF within a reasonable time 
(a few hours). The neutron DPDF is a powerful tool, and was used in observing dynamic dielectric polarization in relaxor ferroelectrics (Fig. 4) [20]. We are currently using this method in determining the local dynamics in liquids, including superfluid ${ }^{4} \mathrm{He}$. The Van Hove function has been known for a long time, but it was used only once in the experiment to observe the local phonon mode in water [23]. We are using this function to describe the elementary excitations in high-temperature liquid. It is a powerful way to describe overdamped local excitations, and is likely to be used more often in the future.

\section{Describing Local Structure}

Most properties in liquids and solids are determined by more collective interaction, whereas the structural information provided by scattering measurement represents only the twobody correlation. In order to explain these properties in terms of the pair-correlation alone, a lot of assumptions have to be made, many of which are dubious in nature. Instead, it is useful to consider local structure defined by an atom and its nearest neighbors. The first and second peaks of the PDF are well separated with a deep minimum in-between, even for liquids and glasses. This allows defining the nearest neighbors using the PDF minimum as a cut-off distance. An alternative way to define the neighbors is through the Voronoi tessellation analysis $[24,25]$. Once the nearest neighbors are specified we can consider atomic connectivity, by defining connectivity between atoms which are the nearest neighbors to each other. The way atoms are connected is called topology of the structure. Even though the local structure cannot be directly determined by diffraction, by building a model by simulation and comparing the PDF of the model with the PDF determined by diffraction to justify the model, we can gain some insight about the nature of the local structure in glasses and liquids.

The importance of focusing on the topology of atomic connectivity is seen in the recent discovery that the dynamics of the atomic connectivity network is directly related to the viscosity of a high-temperature liquid [19]. Even though viscosity is one of the most fundamental properties of a liquid, its atomistic origin has not been known. Through simulation we found that the action of cutting and forming the atomic bond, or action of changing the nearest neighbor, is directly controlling viscosity. Thus instead of phonons this cutting and forming the atomic bond, 
the action of changing the local topology, is the elementary excitation in liquids. We named this excitation "anankeon" after the Greek Goddess of force, constraint and destiny [26].

Examination of the topology of the Voronoi polyhedra [24,25] led to an important observation that they are dominated by a pentagonal face, which is rarely found in crystals because five-fold symmetry is incompatible with translational symmetry. Frank [27] suggested that an icosahedral cluster, which has dominant five-fold symmetry and is stable because it is a tightly packed structure with its surface all covered by triangles, may be found in liquids but not in crystals, and it explains the stability of a liquid allowing for deep supercooling. Indeed icosahedral clusters are frequently found in liquids and glasses [28].

Icosahedral clusters in liquids and glasses, however, are always distorted and do not have perfect symmetry [29]. This is because the atomic clusters embedded in the glassy matrix do not nicely fit to the matrix. The Voronoi polyhedral analysis tells us only the topology of atomic connectivity, not the degree of distortion. The PDF tells us the bond distortion in terms of the distribution of the interatomic distances, but only the distortion in the two-body correlation. The concept of the atomic-level stress [30] was introduced to bridge the two. The atomic-level stress tensor on the $i$-th atom is defined by

$$
\sigma_{i}^{\alpha \beta}=\frac{1}{V_{i}} \sum_{j} f_{i j}^{\alpha} r_{i j}^{\beta}
$$

where $\alpha$ and $\beta$ denote the Cartesian coordinates, $V_{i}$ is the local volume of atom $i, f_{i j}$ is the twobody force acting on the atom $i$ due to the atom $j$, and $r_{i j}$ is the separation between atoms $i$ and $j$ [30]. Its trace defines the atomic-level pressure,

$$
p_{i}=\frac{\sigma_{i}^{x x}+\sigma_{i}^{y y}+\sigma_{i}^{z z}}{3}
$$

while five other components or the combinations of the components define the shear stresses.

The atomic-level stress is created by the geometrical mismatch between the atomic size and the size of the hole in which the atom is placed [31]. In the case of an icosahedral cluster, the distance between the vertex and the center is shorter by 5\% than the distance between vertices. Thus if the cluster is formed without the central atom and by all the peripheral atoms touching each other and perfectly maintaining the symmetry, the space for the central atom to 
enter is smaller than the atom by $10 \%$ in radius. Then in order to insert the central atom into the hole without deforming the cluster the volume of the inserted atom has to be reduced by $27 \%$ (= $\left.1-(0.9)^{3}\right)$. Thus if we forcibly place the center atom, this atom will be under a compressive stress.

In general the atomic-level stresses are closely related to the local topology. For instance, the atomic-level pressure is linearly related to the number of nearest neighbors, the coordination number [31]. This is because the size and shape of the hole in the Voronoi polyhedron is directly determined by the topology just as in the case of the icosahedral cluster. A large coordination number will result in a larger hole in the cluster. In a monoatomic system the ideal coordination number happens to be around $4 \pi=12.56$ [32], suggesting that a 12 coordinated icosahedron is close to an ideal, but not really ideal, local cluster. In this way the concept of the atomic-level stresses nicely connects the topology of atomic connectivity to physical properties, such as energy $[31,33]$.

It was found that the elastic self-energy of the atomic-level stress obeys the equipartition theorem in high-temperature liquids [31,34,35];

$$
\frac{V}{2 B}\left\langle p^{2}\right\rangle=\frac{V}{2 G}\left\langle\sigma_{s}^{2}\right\rangle=\frac{k T}{4}
$$

where $\left\langle\ldots\right.$. > represents thermal and ensemble average, $\sigma_{s}$ is one of the five shear stresses, $V$ is atomic volume, $B$ is bulk modulus and $G$ is shear modulus. The sum of the self-energies of the six stress components adds up to $(3 / 2) k T$, the total potential energy of a harmonic oscillator in three-dimensions. When a liquid is frozen into a glass the stress fluctuation at $T_{f}$ remains in the glass. Therefore the value of $\left\langle p^{2}\right\rangle$ is a good measure of $T_{f}$. Indeed the change in the PDF during structure relaxation [12] is nicely explained in terms of the reduction in $\left\langle p^{2}\right\rangle$ [36], therefore the reduction in $T_{f}$. This enabled the determination in the increase in $T_{f}$, rejuvenation, during mechanical deformation of a metallic glass [14]. Furthermore the glass transition temperature, $T_{g}$, can be calculated from a similar consideration involving the universal critical volume strain at the glass transition and the effect of the Poisson's ratio (Fig. 5) [37].

\section{Disorder in Nuclear Materials}


Nuclear materials often suffer from irradiation damages during the operation of a nuclear reactor. Irradiation damages on crystalline materials produce lattice defects such as vacancies and interstitials by the impact of irradiating particles. However, much of them disappear in a relatively short time due to recombination of vacancies and interstitials and coagulation of vacancies to form voids and cavities. Irradiation also locally melts the lattice for a short time because of the energy deposited by the particles. Diffraction measurements cannot catch such fast incidents of transient damage and local melting, because they detect the structure averaged over space and time. The problem is not the timescale of measurement, but the fact that the volume fraction of the damaged region at an instant is too small to detect. They can only detect lasting structural defects present long after irradiation. Nevertheless these long-lasting defects are more damaging to the performance of the nuclear material, and need to be carefully evaluated.

On the other hand quantifying disorder in a material can be very important in developing new nuclear materials. For instance, glasses are relatively irradiation resistant because they are already disordered in the structure [38,39]. Also materials with high degrees of disorder could be useful as a nuclear material. Irradiation creates lattice defects, and the structure becomes amorphous when the local atomic-level strain reaches the critical value [40]. Compositional disorder in alloys induces strong local distortion in the lattice [41]. Therefore alloys become amorphized with less irradiation [40], and amorphization and local melting wipes away much of lattice defects [42]. For this reason multi-component alloys, such as the high-entry alloys [4345], are promising as irradiation resistant nuclear materials [46-48].

\section{Conclusion}

Extensive structural disorder is often found in nuclear materials, usually due to irradiation damage by neutrons and other particles. However, characterization of structural disorder through diffraction experiment is an involved endeavor which requires much more effort compared to characterization of crystalline solids. Nevertheless, thanks to the advent of the synchrotronbased radiation sources, such as synchrotron x-ray radiation sources and pulsed neutron sources which provide intense high-energy scattering probes, accurate measurements of the static and 
dynamic pair-density functions became relatively easier. It is now possible to quantify the degree of disorder through diffraction measurements, for instance in terms of the fictive temperature, and elucidate various properties. In addition materials with strong disorder, such as

glasses and high-entropy alloys, are likely to be candidates as new nuclear materials owing to their high irradiation resistance. Characterization of disorder will continue to be an important activity in the field of nuclear materials research.

\section{Acknowledgments}

This work was supported by the U.S. Department of Energy, Office of Sciences, Basic Energy Sciences, Materials Science and Engineering Division.

\section{References}

1. A. Q. Tool, J. Amer. Cer. Soc. 29, 240 (1946).

2. G. W. Scherer, Relaxation in Glass and Composites (Wiley, New York, 1986).

3. B. E. Warren, X-ray Diffraction (Dover, New York, 1969, 1990).

4. T. Egami and S. J. L. Billinge, Underneath the Bragg Peaks: Structural Analysis of Complex Materials (Pergamon Press, Elsevier Ltd., Oxford, 2003; 2012).

5. T. Egami, in Bulk Metallic Glasses, ed. M. Miller and P. Liaw (Springer-Verlag, Berlin, 2008), p. 27.

6. P. J. Chupas, X. Qiu, J. C. hanson, P. L. Lee, C. P. Grey and S. J. L. Billinge, J. Appl. Cryst. 36, 1342 (2003).

7. X. Qiu, J. W. Thompson and S. J. L. Billinge, J. Appl. Cryst. 37, 678 (2004).

8. P. F. Peterson, M. Gutmann, T. Proffen and S. J. L. Billinge, J. Appl. Cryst. 33, 1192 (2000); http://pdfgetn.sourceforge.net/.

9. E. A. Stern, D. E. Sayers and F. W. Lytle, Phys. Rev. B 11, 4836 (1975).

10. F. W. Lytle, D. E. Sayers and E. A. Stern, Phys. Rev. B 11, 4825 (1975).

11. J.-H. Chung, T. Proffen, S.-I. Shamoto, A. M. Ghorayeb, L. Croguennec, W. Tian, B. C. Sales, R. Jin, D. Mandrus and T. Egami, Phys. Rev. B, 71, 064410 (2005).

12. T. Egami, J. Mat. Sci. 13, 2587 (1978). 
13. W. Dmowski, C. Fan, M. L. Morrison, P. K. Liaw, and T. Egami, Mater. Sci. Eng. A, 471, 125-129 (2007).

14. Y. Tong, T. Iwashita, W. Dmowski, H. Bei, Y. Yokoyama and T. Egami, Acta Mater. 86, 240 (2015).

15. Y. Suzuki, J. Haimovich and T. Egami, Phys. Rev. B 35, 2162 (1987).

16. W. Dmowski, T. Iwashita, C.-P. Chuang, J. Almer and T. Egami, Phys. Rev. Lett. 105, 205502 (2010).

17. W. Dmowski, Y. Tong, T. Iwashita, Y. Yokoyama and T. Egami, Phys. Rev. B, 91, 060101(R) (2015).

18. S. W. Lovesey, Theory of Neutron Scattering from Condensed Matter (Oxford Science, Oxford, 1984).

19. T. Iwashita, D. M. Nicholson and T. Egami, Phys. Rev. Lett., 110, 205504 (2013).

20. W. Dmowski, S. B. Vakhrushev, I.-K. Jeong, M. P. Hehlen, F. Trouw and T. Egami, Phys. Rev. Lett., 100, 137602 (2008).

21. T. Egami and W. Dmowski, Z. Kristallogr., 227, 233 (2012).

22. L. Van Hove, Phys. Rev. 95, 249 (1954).

23. P. Abbamonte, K. D. Finkelstein, M. D. Collins and S. M. Gruner, Phys. Rev. Lett. 92, 237401 (2004).

24. J. D. Bernal, Nature 183, 141 (1959).

25. J. L. Finney, Proc. Roy. Soc. London, A 319, 479 (1970).

26. T. Egami, Mod. Phys. Lett. B, 28, 1430006 (2014).

27. F. C. Frank, Proc. Roy. Soc. London, A 215, 43 (1952).

28. K. F. Kelton, G. W. Lee, A. K. Gangopadhyay, R. W. Hyers, T. J. Rathz, J. R. Rogers, M. B. Robinson and D. S. Robinson, Phys. Rev. Lett. 90, 195504 (2003).

29. A. Hirata, L. J. Kang, T. Fujita, B. Klumov, K. Matsue, M. Kotani, A. R. Yavari and M. W. Chen, Science, 341, 376 (2013).

30. T. Egami, K. Maeda, and V. Vitek, Phil. Mag. A 41, 883 (1980).

31. T. Egami, Progr. Mater. Sci. 56, 637 (2011).

32. T. Egami and S. Aur, J. Non-Cryst. Solids 89, 60 (1987).

33. T. Egami and D. Srolovitz, J. Phys. F 12, 2141 (1982).

34. S.-P. Chen, T. Egami and V. Vitek, Phys. Rev. B 37, 2440 (1988). 
35. V. A. Levashov, R. S. Aga, J. R. Morris and T. Egami, Phys. Rev. B, 78, 064205 (2008).

36. D. Srolovitz, T. Egami, and V. Vitek, Phys. Rev. B 24, 6936 (1981).

37. T. Egami, S. J. Poon, Z. Zhang and V. Keppens, Phys. Rev. B, 76, 024203 (2007).

38. E. A. Kramer and W. L. Johnson, Appl. Phys. Lett. 35, 815 (1979).

39. D. J. Magagnosc, R. Ehrbar, G. Kumar, M. R. He, J. Schroers and D. S. Gianola, Scientific Reports 3, 1096 (2013).

40. W. Guo, T. Iwashita and T. Egami, Acta Mater., 68, 229 (2014).

41. W. Guo, W. Dmowski, Ph. Rack, P. Liaw, and T. Egami, Metall. Mater. Trans. A, 44, 1994 (2013).

42. T. Egami, W. Guo, P. D. Rack and T. Nagase, Met. Mat. Trans. A, 45, 180 (2014).

43. B. Cantor, I. T. H. Chang, P. Knight and A. J. B. Vincent, Mater. Sci. Eng. A, 375-377, 213 (2004).

44. J.-W. Yeh, S.-K. Chen, S.-J. Lin, J.-Y. Gan, T.-S. Chin, T.-T. Shun, C.-H. Tsau, and S.-Y. Chang: Adv. Eng. Mater. 6, 299 (2004).

45. C.-Y. Hsu, J.-W. Yeh, S.-K. Chen and T.-T. Shun, Metal. Mater. Trans. A, 35, 1465 (2004).

46. T. Nagase, S. Anada, P.D. Rack, J.H. Noh, H. Yasuda, H. Mori, and T. Egami: Intermetallics, 26, 122 (2012).

47. T. Nagase, S. Anada, P.D. Rack, J.H. Noh, H. Yasuda, H. Mori, and T. Egami: Intermetallics, 38, 70 (2013).

48. T. Nagase, P. D. Rack and T. Egami, Intermetallics, 59, 32 (2015). 


\section{Figure Captions:}

Fig. 1 (a) Structure function, $S(Q)$, and (b) PDF, $g(r)$, of amorphous $\mathrm{Zr}-\mathrm{Cu}-\mathrm{Ni}-\mathrm{Al}-\mathrm{Ti}$ alloy by xray and neutron diffraction [5].

Fig. 2 PDF of $\mathrm{LiNiO}_{2}$ determined by pulsed neutron diffraction at 10 and $585 \mathrm{~K}$, (a) up to 200 $\AA$, (b) $1-7 \AA$, and (c) $165-185 \AA$ [11]. Note that at short distances (b) the PDF at $585 \mathrm{~K}$ has lower peaks due to thermal excitation, whereas at large distances (c) the opposite is the case because of the formation of domains with the size $\sim 90 \AA$.

Fig. 3 (Above) Isotropic PDF, $g_{0}^{0}(r)$, and (below) anisotropic PDF, $g_{2}^{0}(r)$, of glassy $\mathrm{Zr}-\mathrm{Cu}-\mathrm{Ni}$ Al-Ti alloy under uniaxial stress at room temperature [16].

Fig. 4 Dynamic PDF of $\mathrm{Pb}\left(\mathrm{Mg}_{1 / 3} \mathrm{Nb}_{2 / 3}\right) \mathrm{O}_{3}$ at $450 \mathrm{~K}$. The diffuse signal at 1.5 and $3.5 \AA$ above $20 \mathrm{meV}$ indicate dynamic polarization [20].

Fig. 5 Values of $k T_{g} / 2 B V$, where $T_{g}$ is the glass transition temperature, $B$ is bulk modulus and $V$ is atomic volume, plotted against the Poisson's ratio. Circles are the experimental values and the line indicates the theory [37]. 


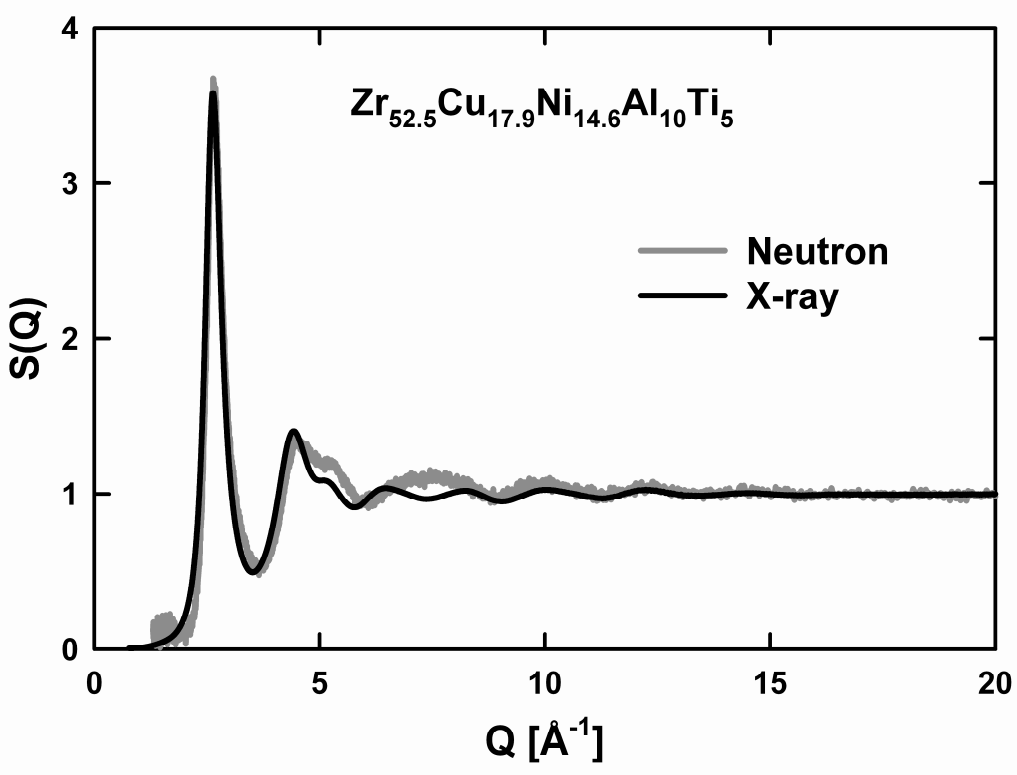

(a)

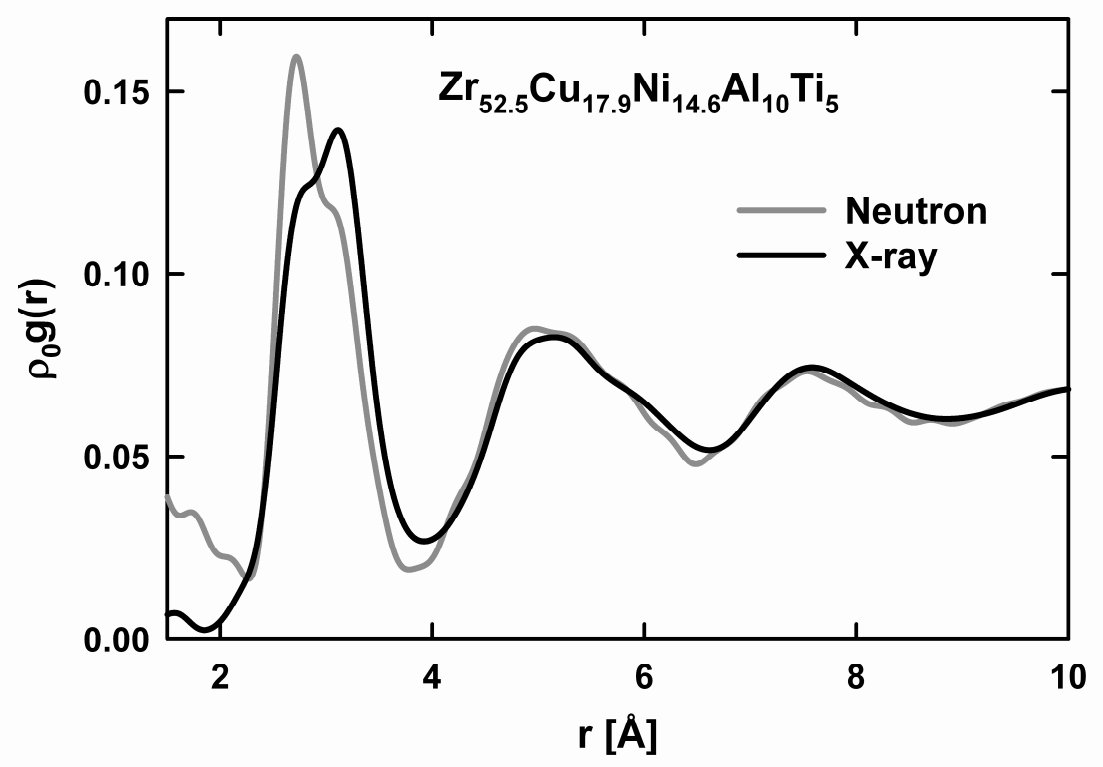

(b)

Fig. 1 


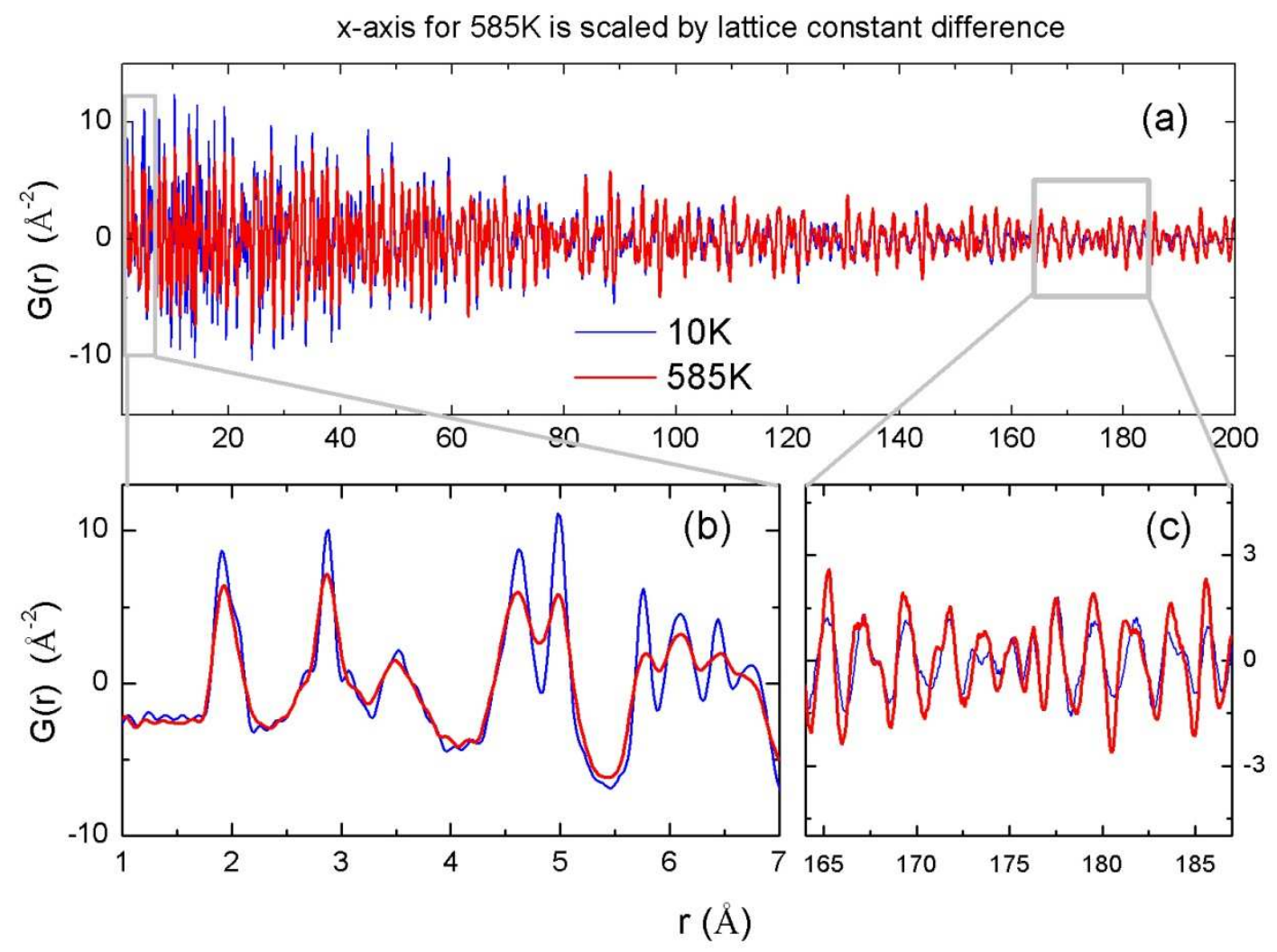

Fig. 2 


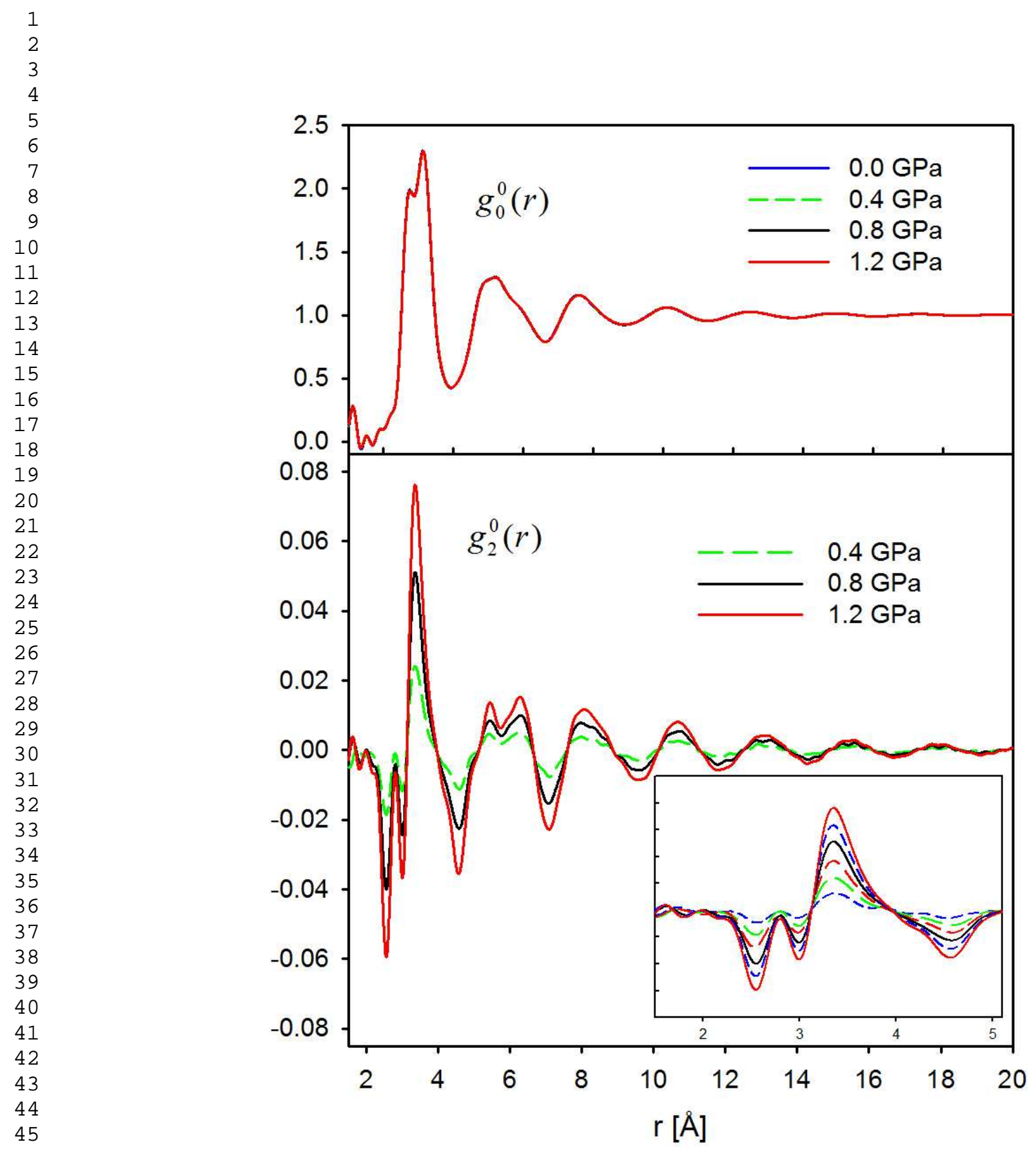

46

47

48

49

50

51

52

53

54

55

56

57

58

59

60

61

62

Fig. 3 


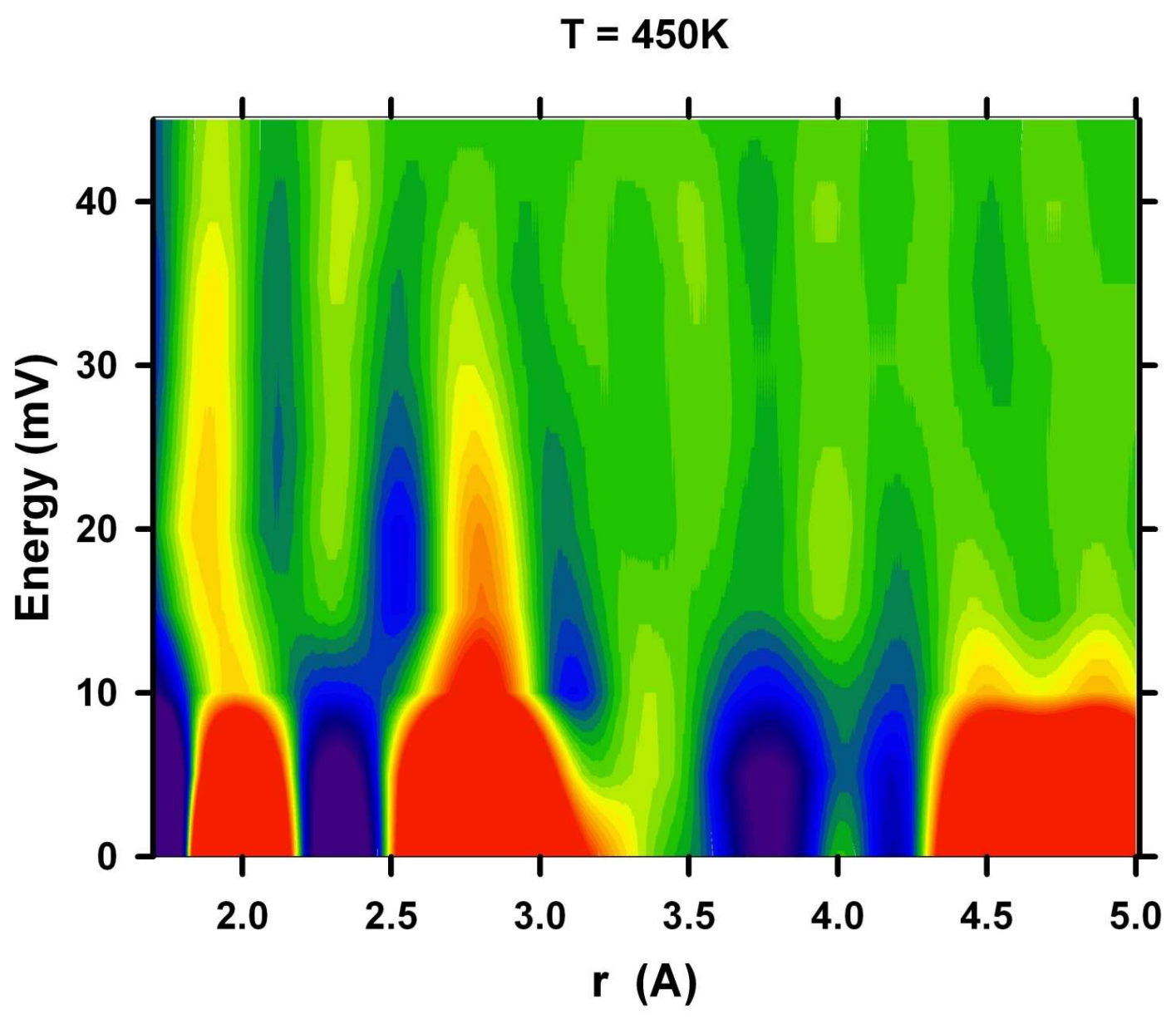

Fig. 4 


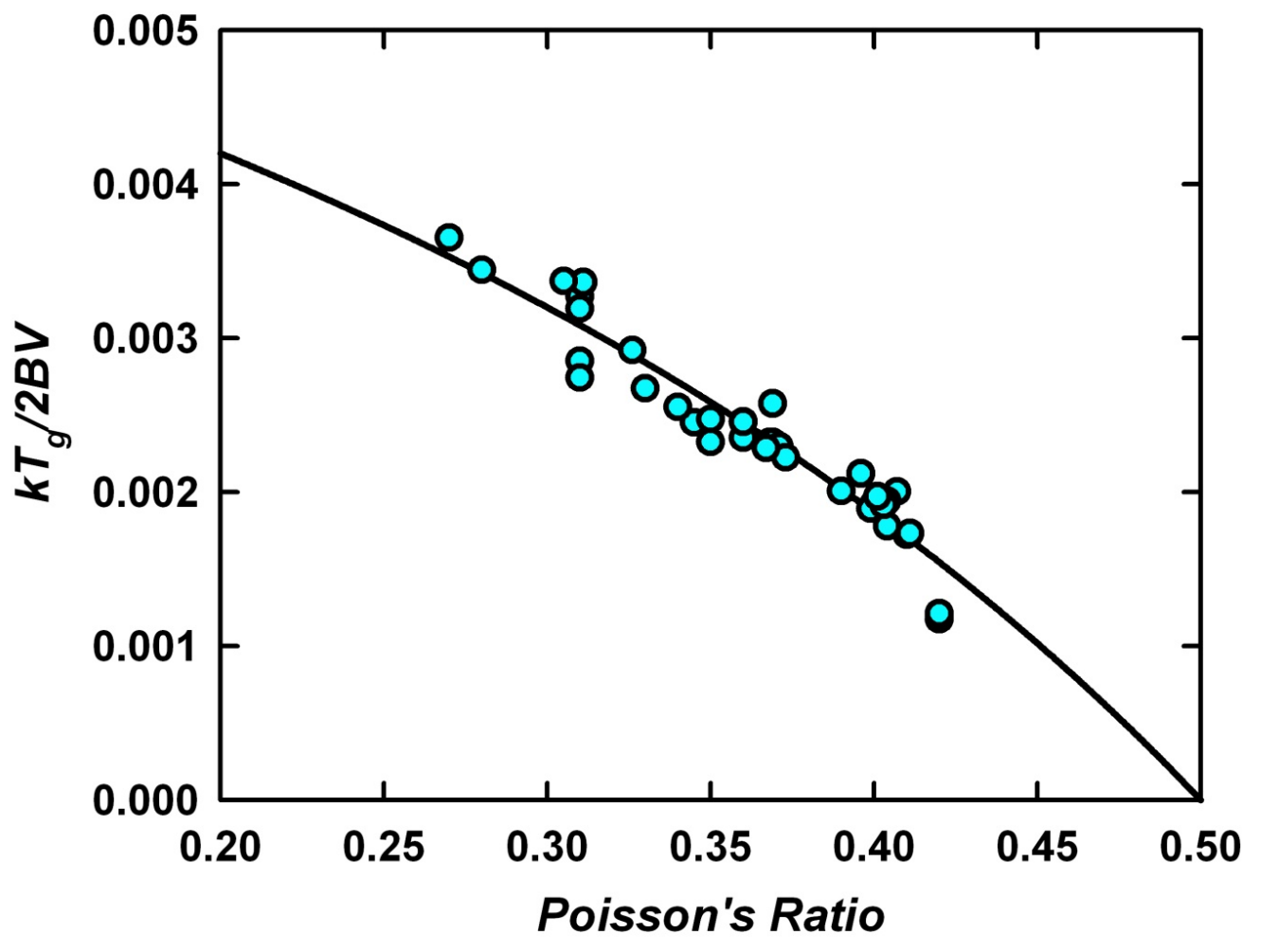

Fig. 5 